\title{
Desenvolvimento e avaliação de um agente conversacional para candidatos à doação de sangue
}

\author{
Mateus Klein Roman ${ }^{1}$, Ericles Andrei Bellei ${ }^{2}$, Daiana Biduski ${ }^{2}$ \\ Cristiane da Silva R. de Araújo ${ }^{3}$, Ana Carolina B. De Marchi ${ }^{1,2}$ \\ ${ }^{1}$ Curso de Ciência da Computação - Universidade de Passo Fundo (UPF) \\ ${ }^{2}$ Programa de Pós-Graduação em Computação Aplicada - UPF \\ ${ }^{3}$ Serviço de Hemoterapia do Hospital São Vicente de Paulo - Passo Fundo (HSVP) \\ \{152086, 168729, 130011, crisrodrigues, carolina\}@upf.br

\begin{abstract}
Resumo. A captação de doadores de sangue é um ato importante nos serviços de hemoterapia e hemocentros. Entretanto, o desconhecimento sobre o processo de doação ainda é uma barreira. Este estudo apresenta o desenvolvimento e a avaliação de um agente conversacional na plataforma Google Assistente para responder perguntas e explicar o processo de doação a potenciais doadores. Foi conduzida uma avaliação de experiência do usuário com 50 participantes que interagiram com o agente. Os resultados revelaram níveis positivos para a experiência de uso, sugerindo o potencial do agente conversacional como um ferramenta de disseminação de informação.
\end{abstract}

\begin{abstract}
Recruiting blood donors is an important act in the blood centers. However, insufficient knowledge and misconceptions about the donation process are still common. This study presents the development and evaluation of a conversational agent to answer questions and explain the donation process to potential blood donors. A user experience assessment was performed with 50 participants who interacted with the agent. The results revealed positive and satisfactory user experience, suggesting the agent as a creative and captivating tool for information outreach.
\end{abstract}

\section{Caracterização do problema e motivação}

$\mathrm{O}$ ato de doar sangue é um gesto de solidariedade e também considerado uma preocupação mundial, pois não há substância que possa substituir o sangue [Rodrigues and Reibnitz 2011]. Segundo o [Ministério da Saude 2018], em 2018, apenas 1,6\% da população era doadora de sangue, enquanto a Organização Mundial da Saúde preconiza que $5 \%$ da população faça doação para suprir a demanda transfusional. Muitos doadores considerados aptos a fazer uma doação de sangue enfrentam problemas como a falta de informação ou informações inexatas sobre o processo de doação de sangue. Isso acaba dificultando a concretização da doação de sangue e agravando os problemas de estoques baixos [Carlesso et al. 2017].

A tecnologia e a comunicação podem se tornar aliadas na criação de ferramentas de difusão de informações e esclarecimento de dúvidas para os candidatos à doação de 
sangue. Com a crescente utilização e o aprimoramento de ferramentas que fazem processamento em linguagem natural, os agentes conversacionais, por vezes chamados de chatbots, podem ajudar no processo de difusão de informações, de maneira fácil e engajadora [Laranjo et al. 2018, Biduski et al. 2020]. Nesse cenário, este trabalho teve o objetivo de desenvolver e a avaliar um agente projetado para esclarecer as principais dúvidas sobre o processo de doação de sangue a potenciais doadores.

\section{Trabalhos relacionados}

São ainda incipientes as iniciativas relacionadas ao uso de tecnologia para auxiliar na difusão de informações relevantes sobre doação de sangue. Entre os projetos disponíveis, [Brislin et al. 2017] criaram um aplicativo para localizar doadores de sangue geograficamente próximos do usuário. Por sua vez, [Dutta et al. 2018] desenvolveram um sistema web para gerenciar campanhas de doação de sangue, nas quais os doadores podem planejar as atividades da campanha de acordo com suas preferências. $\mathrm{O}$ [Canadian Blood Services 2017] desenvolveu um chatbot para envolver jovens doadores no Facebook Messenger, ajudando a aprender mais sobre o processo de doação e sobre as pessoas que precisam. Em Copenhague, uma empresa criou uma plataforma chamada BloodLink [Mayroth 2017], com o objetivo de conectar doadores, bancos de sangue e hospitais para minimizar o desperdício de sangue. Outra solução desenvolvida nesse domínio é o Donor Finder, um chatbot que permite aos usuários solicitar e doar sangue a outros usuários próximos [Paul 2017]. Enquanto isso, o agente conversacional apresentado neste trabalho está entra as primeiras iniciativas semelhantes que abordam o desenvolvimento e o uso dessas soluções no Brasil.

\section{Desenvolvimento do agente conversacional}

Para este projeto [Roman et al. 2020], iniciou-se com a criação da base de conhecimento e o planejamento dos diálogos. Sua construção foi feita juntamente com profissionais do Serviço de Hemoterapia do Hospital São Vicente de Paulo (SHHSVP). A base abrangeu informações sobre as etapas mais importantes da doação de sangue, as perguntas mais frequentes dos candidatos à doação na recepção do serviço, e cartilhas explicativas do SHHSVP já desenvolvidas e validadas sobre o assunto.

O agente conversacional foi implementado com o motor de programação e hospedagem Dialogflow [Google 2019]. Esse motor é acessível em uma interface web e permite a programação de detecção de intenções, contextos, entidades e disparo de ações de resposta e sugestões de continuidade de diálogo, interpretando as variáveis em linguagem natural. Cada intenção está associada a um conjunto de frases de treinamento, que foram definidas durante o andamento do projeto, além de ações, respostas e contextos. Para o agente conversacional abordar aspectos mais amplos sobre a o processo da doação de sangue, programou-se 30 intenções sobre o processo de doação de sangue (Tabela 1).

O agente conversacional está disponível na plataforma Google Assistente e pode ser acessado com a frase de invocação “Ok Google, falar com Hemoterapia HSVP”. Após invocado, o agente é instanciado e inicia o diálogo. O usuário pode interagir de maneira multimodal digitando, falando e clicando nos botões da interface. $\mathrm{O}$ agente sempre responde exibindo informações visuais e também pode falar, caso seu interlocutor utilize a fala durante o diálogo. Futuramente, o agente poderá ser adaptado e implantado em outras redes sociais, conforme a disponibilidade de integrações disponíveis no Dialogflow. 
No primeiro estágio do diálogo, o agente apresenta-se como o personagem Hemozito, o mascote do SHHSVP. Depois, o agente lista os assuntos sobre os quais está apto a falar sobre, incentivando o usuário a dar continuidade no diálogo. Os dois passos iniciais de interação e duas intenções que o agente pode responder estão ilustrados na Figura 1. Durante todo o restante da interação, o usuário pode perguntar livremente, falar e selecionar os botões que aparecem como sugestões.

\section{Tabela 1. Tópicos e questões sobre doação de sangue que foram treinadas para} a base de conhecimento do agente conversacional.

Quem pode doar sangue?

Como doar sangue?

Quais são as etapas de doação de sangue?

Qual é o intervalo de doação de sangue?

Quem não pode doar sangue?

Qual a quantidade de sangue coletada?

Quais são os exames laboratoriais realizados?

Quais as recomendações para o dia da doação?

Sobre a Hemoterapia HSVP

Qual é o horário de atendimento?

Preciso agendar para doar sangue?

Quem é tatuado pode doar sangue?

Quem fez micropigmentação pode doar?

Qual o telefone do serviço de hemoterapia?

Vou fazer uma doação de sangue!
Quem fez cirurgia pode doar?

Preciso doar no nome de alguém?

Recebi transfusão, quantas doações preciso fazer?

Qual é o sangue mais importante?

O que você pode responder?

Preciso doar sempre?

Doar sangue dói?

Preciso doar em jejum?

Posso doar sangue sem dormir?

Tenho diabetes, posso doar?

Tenho problema cardíaco, posso doar?

Tomo remédio para a tireóide, posso doar?

Tomo remédio, posso doar sangue?

Qual a localização do serviço de hemoterapia?

Terminar a conversa
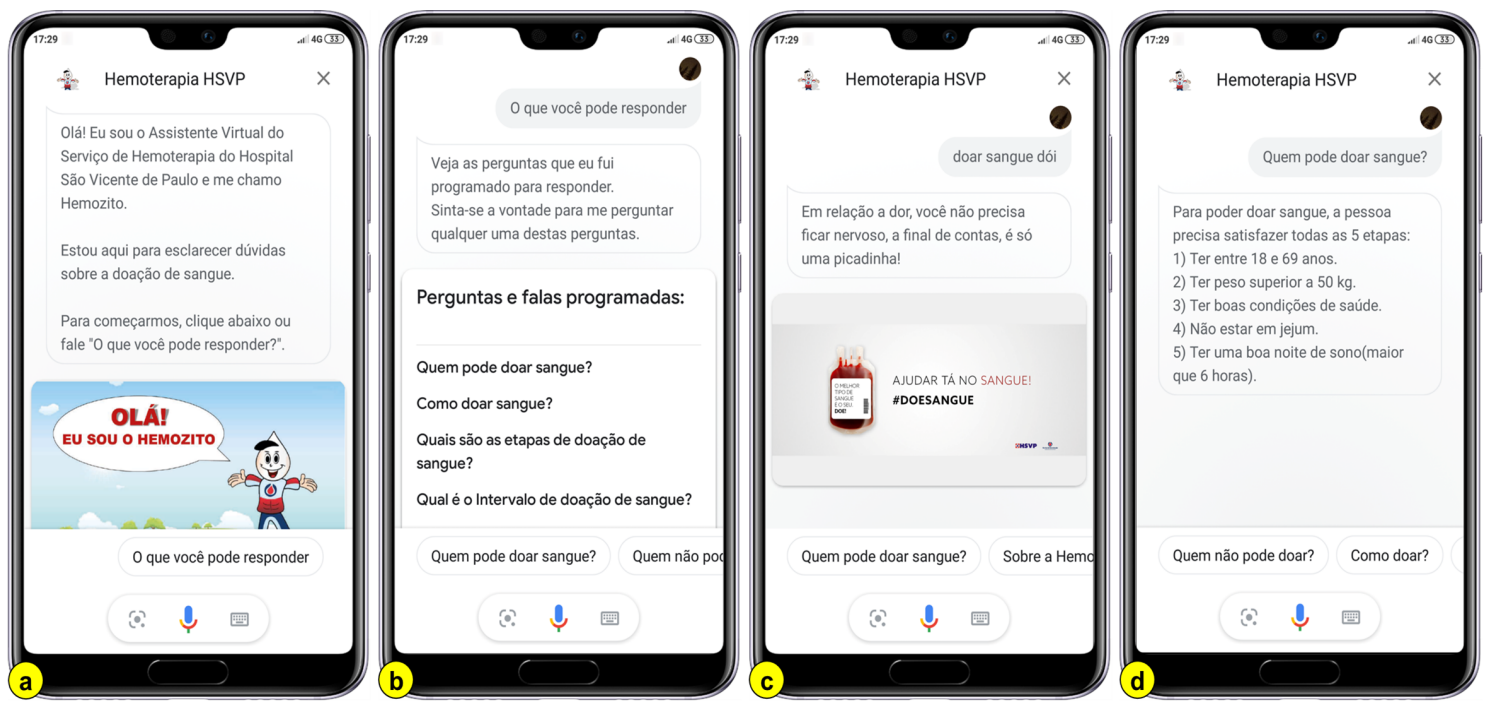

Figura 1. Exemplo de fluxo de diálogo com o agente conversacional.

\section{Avaliação do agente conversacional}

A avaliação do agente conversacional começou pela aprovação dos procedimentos, envolvendo os participantes da pesquisa, pelo Comitê de Ética da Universidade de Passo Fundo, sob o parecer número 20468919.3.0000.5342, além da aprovação pela Comissão de Pesquisa e Pós-Graduação do Hospital São Vicente de Paulo. Por amostragem não probabilística por conveniência, foram selecionadas 50 pessoas, das quais 16 homens e 34 mulheres, com idades entre 19 e 65 anos $(28 \pm 9,97)$, sendo o único critério de inclusão o participante ter aptidão em lidar com smartphone. 
Além de um questionário sociodemográfico para caracterização da amostra, a avaliação contemplou 2 instrumentos de avaliação. O primeiro instrumento foi o questionário UEQ (User Experience Questionnaire) [Laugwitz et al. 2008], utilizado para coletar uma impressão geral sobre a experiência do usuário (UX) após a interação. Esse questionário abrange 26 perguntas objetivas, em escala de diferencial semântico, sobre aspectos de usabilidade (eficiência, perspicácia, confiabilidade) e aspectos de experiência (originalidade, estimulação). Por fim, o segundo instrumento foi um questionário com três perguntas discursivas, elaborada pelos autores, para o participante relatar sua experiência de interação: Como foi a sua experiência interagindo com o agente conversacional? Você utilizaria novamente o agente conversacional e porquê? Você recomendaria a utilização do agente conversacional para outras pessoas e porquê?

O procedimento de coleta de dados foi dividido em 4 etapas. Na primeira, cada participante foi individualmente instruído sobre os objetivos da avaliação e assinou o termo de consentimento livre e esclarecido. Na segunda, o participante respondeu ao questionário de caracterização de amostra. Na terceira etapa, o avaliador forneceu um smartphone e orientou o participante sobre o funcionamento do agente conversacional. Cada participante fez uma interação livremente com o agente conversacional por um tempo de 6 minutos utilizando voz, texto e elementos visuais. Após a interação, na quarta etapa, o participante respondeu ao questionário UEQ e às perguntas discursivas.

Os dados das respostas do questionário UEQ foram avaliados com a ferramenta de análise própria do UEQ [Schrepp et al. 2014]. Os resultados produzidos (Tabela 2) sumarizam médias dos scores de todos os aspectos da experiência do usuário teorizados no UEQ. Os scores são representados em escala de -3.0 a 3.0, cujos valores inferiores a -0.8 representam resultados de avaliação insatisfatórios, valores entre -0.8 e 0.8 apontam resultados neutros, e valores superiores a 0.8 indicam resultados satisfatórios.

Em relação à média das avaliações individuais das perguntas do UEQ (Tabela 2), todas as avaliações tiveram um resultado excelente (superior a 0.8). As questões relacionadas a tédio e previsibilidade tiveram os valores inferiores que as demais, mas ainda assim caracterizadas como excelentes. Em relação aos dados que ficaram acima do esperado, destaca-se interesse, bondade, eficiência, praticidade e organização, juntamente das escalas de qualidade.

Respondendo às perguntas descritivas, todos os participantes expressaram uma experiência positiva e satisfatória. A maioria das opiniões mencionou que o agente era interessante e inovador. Outros termos mencionados pelos participantes reportaram o agente como divertido, esclarecedor, útil, intuitivo, de fácil uso, e rápido para obter informações. Todos os participantes também declararam que usariam o agente novamente e o recomendariam a outros. As justificativas para isso eram análogas em ambas as perguntas e se referiam ao conteúdo do agente, ao estilo de interação e às implicações e benefícios percebidos. Em relação ao conteúdo, o agente foi considerado interessante por apresentar informações em etapas, de maneira didática e cativante, incluindo recursos diversificados de texto, imagem e voz. Por fim, alguns participantes relataram que o agente conversacional pode incentivar um potencial doador, explicando a importância de doar sangue e apresentando todas as principais informações necessárias para isso. 
Tabela 2. Resultados dos itens e escalas do User Experience Questionnaire.

\begin{tabular}{|c|c|c|c|}
\hline Classificação & Média* \pm DP & IC $(95 \%)$ & Escala / Qualidade \\
\hline Desagradável/Agradável & $2.600 \pm 0.670$ & 0.186 & Atratividade \\
\hline Incompreensível/Compreensível & $2.640 \pm 0.663$ & 0.184 & Transparência \\
\hline Criativo/Sem criatividade & $2.480 \pm 1.129$ & 0.313 & Inovação \\
\hline De Fácil aprendizagem/De difícil aprendizagem & $2.520 \pm 1.035$ & 0.287 & Transparência \\
\hline Valioso/Sem valor & $2.460 \pm 1.182$ & 0.328 & Estimulação \\
\hline Entediante/Excitante & $1.500 \pm 1.488$ & 0.412 & Estimulação \\
\hline Desinteressante/Interessante & $2.660 \pm 0.519$ & 0.144 & Estimulação \\
\hline Imprevisível/Previsível & $1.340 \pm 1.698$ & 0.471 & Controle \\
\hline Rápido/Lento & $2.520 \pm 0.646$ & 0.179 & Eficiência \\
\hline Original/Convencional & $2.180 \pm 1.335$ & 0.370 & Inovação \\
\hline Obstrutivo/Condutor & $2.220 \pm 1.055$ & 0.293 & Controle \\
\hline Bom/Mau & $2.800 \pm 0.452$ & 0.125 & Atratividade \\
\hline Complicado/Fácil & $2.440 \pm 0.929$ & 0.258 & Transparência \\
\hline Desinteressante/Atrativo & $2.600 \pm 0.670$ & 0.186 & Atratividade \\
\hline Comum/Contemporâneo & $2.020 \pm 1.491$ & 0.413 & Inovação \\
\hline Incômodo/Cômodo & $2.400 \pm 0.808$ & 0.224 & Atratividade \\
\hline Seguro/Inseguro & $2.440 \pm 1.128$ & 0.313 & Controle \\
\hline Motivante/Desmotivante & $2.360 \pm 0.921$ & 0.255 & Estimulação \\
\hline Atende as expectativas/Não atende as expectativas & $2.600 \pm 1.010$ & 0.280 & Controle \\
\hline Ineficiente/Eficiente & $2.740 \pm 0.487$ & 0.135 & Eficiência \\
\hline Evidente/Confuso & $2.560 \pm 0.577$ & 0.160 & Transparência \\
\hline Impraticável/Prático & $2.680 \pm 0.513$ & 0.142 & Eficiência \\
\hline Organizado/Desorganizado & $2.660 \pm 0.593$ & 0.164 & Eficiência \\
\hline Atraente/Feio & $2.380 \pm 0.855$ & 0.237 & Atratividade \\
\hline Simpático/Antipático & $2.420 \pm 0.758$ & 0.210 & Atratividade \\
\hline Conservador/Inovador & $2.380 \pm 1.276$ & 0.354 & Inovação \\
\hline Escala de Atratividade & $2.533 \pm 0.515$ & 0.143 & Atratividade \\
\hline Escala de Transparência & $2.540 \pm 0.585$ & 0.162 & Qualidade Pragmática \\
\hline Escala de Eficiência & $2.650 \pm 0.404$ & 0.112 & Qualidade Pragmática \\
\hline Escala de Controle & $2.150 \pm 0.587$ & 0.163 & Qualidade Pragmática \\
\hline Escala de Estimulação & $2.245 \pm 0.715$ & 0.198 & Qualidade Hedônica \\
\hline Escala de Inovação & $2.265 \pm 0.859$ & 0.238 & Qualidade Hedônica \\
\hline
\end{tabular}

\section{Considerações Finais}

Este estudo apresentou um agente conversacional desenvolvido para explicar dúvidas relacionadas ao processo de doação de sangue. A experiência de uso avaliada foi substancialmente positiva e satisfatória, assim como as opiniões e atitudes dos participantes em relação ao agente conversacional. Uma boa experiência do usuário está diretamente relacionada a uma maior predisposição de uso a longo prazo [Biduski et al. 2020] e, consequentemente, a uma maior possibilidade de conhecimento engajamento com a doação de sangue. Dessa forma, um agente conversacional pode ser considerado uma estratégia criativa e engajadora de transmitir conhecimento e incentivar candidatos à doação de sangue. As descobertas reafirmam o potencial do uso dessa tecnologia, especialmente para fins socialmente relevantes.

\section{Agradecimentos}

Os autores agradecem ao Serviço de Hemoterapia do Hospital São Vicente de Paulo pela apoio no desenvolvimento e divulgação da pesquisa, em especial, às profissionais Bruna Accorsi Machado, Larissa Andrea Schons e Luciana Bertelli Dagostini. Pelas bolsas de pesquisa e apoio financeiro, os autores agradecem a Fundação de Amparo à Pesquisa do Estado do Rio Grande do Sul (FAPERGS), Conselho Nacional de Desenvolvimento Científico e Tecnológico (CNPq) e Fundação Universidade de Passo Fundo (UPF). 


\section{Autoria e produções derivadas}

M.K.R. foi bolsista de iniciação científica FAPERGS orientado por A.C.B.M. com colaboração de E.A.B., D.B., e C.S.R.A. O agente conversacional recebeu registro de software no INPI BR5120200001637. Um artigo com os resultados completos [Roman et al. 2020] foi publicado no Journal of Biomedical Informatics (Qualis A2).

\section{Referências}

Biduski, D., Bellei, E., Rodriguez, J., Zaina, L., and De Marchi, A. (2020). Assessing long-term user experience on a mobile health application through an in-app embedded conversation-based questionnaire. Comput Human Behav, 104:106169.

Brislin, M. R. A., Mayan, J. A., Canessane, R. A., and Hamlin, M. R. A. (2017). Blood donation and life saver app. In 2017 2nd International Conference on Communication and Electronics Systems (ICCES), pages 446-451. IEEE.

Canadian Blood Services (2017). Canadian blood services launches new tool to engage young donors. Disponível em https://bit.ly/2OehRTF . Accesso em 23 dez 2019.

Carlesso, L., dos Santos, C. F., de Fátima da Silva Guimarães, R., da Silva, S. L., Viero, V., Vieira, S. V., and Girardon-Perlini, N. M. O. (2017). Estratégias implementadas em hemocentros para aumento da doação de sangue. Rev Bras Prom Saúde, 30:213-220.

Dutta, L., Maji, G., Ghosh, P., and Sen, S. (2018). An integrated blood donation campaign management system. In Adv Intell Syst Comput, pages 133-143. Springer.

Google (2019). Dialogflow, build natural and rich conversational experiences. Disponível em: https://dialogflow.com. Acesso em 12 nov 2019.

Laranjo, L., Dunn, A. G., Tong, H. L., Kocaballi, A. B., Chen, J., Bashir, R., Surian, D., Gallego, B., Magrabi, F., Lau, A. Y. S., and Coiera, E. (2018). Conversational agents in healthcare: a systematic review. J Am Med Inform Assoc, 25(9):1248-1258.

Laugwitz, B., Held, T., and Schrepp, M. (2008). Construction and evaluation of a user experience questionnaire. In Lect Notes Comput Sci, pages 63-76. Springer.

Mayroth, N. (2017). Meet the chatbot that helps you donate blood - The Hindu. Disponível em https://bit.ly/38W8O1J . Accesso em 23 dez 2019.

Ministério da Saude (2018). Ministério da saúde reforça campanha para incentivar doação de sangue. Disponível em http://www.saude.gov.br/noticias/agencia-saude/44728saude-reforca-campanha-para-incentivar-doacao-de-sangue . Accesso em 23 dez 2019.

Paul, A. (2017). Donor Finder - A Messenger bot allows people to request blood donation from nearby donors. Disponível em https://bit.ly/2RLRff6. Accesso em 23 dez 2019.

Rodrigues, R. S. M. and Reibnitz, K. S. (2011). Estratégias de captação de doadores de sangue: uma revisão integrativa da literatura. Texto \& Contexto - Enfermagem, 20(2).

Roman, M. K., Bellei, E., Biduski, D., Pasqualotti, A., De Araujo, C., and De Marchi, A. (2020). "Hey assistant, how can I become a donor?" The case of a conversational agent designed to engage people in blood donation. J Biomed Inform, 107:103461.

Schrepp, M., Hinderks, A., and Thomaschewski, J. (2014). Applying the user experience questionnaire (UEQ) in different evaluation scenarios. In Design, User Experience, and Usability, pages 383-392. Springer International Publishing. 